\title{
Enhanced Anti-tumor Immune Responses via a Self-assembled Carrier-free Nanovaccine
}

Dan Liu ${ }^{1}$, Bo Deng ${ }^{1}$, Zongran Liu $^{2,3}$, Bing Ma ${ }^{1}$, Xigang Leng ${ }^{1}$, Deling Kong ${ }^{1,4}$, Tianjiao $\mathrm{Ji}^{* 2,3}$, Lanxia Liu*1

1. The Tianjin Key Laboratory of Biomaterials, Institute of Biomedical Engineering, Peking Union Medical College \& Chinese Academy of Medical Sciences, Tianjin 300192, China

2. CAS Key Laboratory for Biomedical Effects of Nanomaterials \& Nanosafety, CAS Center for Excellence in Nanoscience, National Center for Nanoscience and Technology, Beijing 100190, China

3. Center of Materials Science and Optoelectronics Engineering, University of Chinese Academy of Sciences, Beijing 100049, China

4. College of Life Science, Nankai University, Tianjin 300071, China

*Corresponding author

Tianjiao Ji, Ph.D.

CAS Key Laboratory for Biomedical Effects of Nanomaterials \& Nanosafety, CAS Center for Excellence in Nanoscience, National Center for Nanoscience and Technology, Beijing 100190, China

Email: jitj@nanoctr.cn

Lanxia Liu, Ph.D.

Institute of Biomedical Engineering, Peking Union Medical College \& Chinese Academy of Medical Sciences, Tianjin 3000192, China

E-mail: liulanxiabme@163.com 


\section{Materials and Methods}

\section{Nanovaccines self-assembled with the amphiphilic molecule MPLA-CpG}

MPLA-CpG nanoparticles (named as MC NPs) were prepared using amphiphilic molecules conjugated with monophosphatidyl A (MPLA, Sigma-Aldrich Co. MO, USA) and CpG ODN (Type C 2395, sequence: 5'TCGTCGTTTTCGGCGCGCGCCG-3', Sangon Biotech, Shanghai, China) through 1, 1'-Carbonyldiimidazole (CDI, aladdin, Shanghai, China). Briefly, MPLA and CDI were dissolved in DMSO and reacted for 2 hours at room temperature to activate hydroxyl. Then amino modified CpG ODN was mixed with the activated MPLA and reacted for $12 \mathrm{~h}$. Unreacted impurities were removed using a 2-kDa MWCO dialysis tubing. MPLA-CpG molecules could be self-assembled in water into MC NPs, or they mixed with antigens (OVA as a model antigen) at the molar ratio of 4:1, 2:1, 1:1, 1:5, 1:10, respectively, and self-assembled into MPLA-CpG nanoparticle-encapsulated antigens (named as MCO NVs). The results showed that MCO NVs had a relative uniform size when the molar ratio was greater than or equal to $1: 1$. We used the nanovaccine with the molar ratio of $2: 1$ in the following research.

\section{Characterization of nanovaccines}

The conjugation of MPLA and CpG ODN was detected using agarose gel electrophoresis. The size and Zeta potential of nanoparticles were measured with dynamic light scattering (DLS) and Zetasizer Nano ZS (Malvern Instruments Ltd., UK), respectively. Transmission electron microscope (TEM, JEOL JEM-100CX-II, Japan) was performed to observe the morphology of the nanoparticles. To confirm the antigen loading capacity of nanoparticles, MCO NVs were centrifuged at $23000 \times$ rpm for 20 min with Beckman ultracentrifuge (Beckman Coulter, Avanti J-26S XP, USA) and the supernatant was collected to measure the content of OVA using Enhanced BCA Protein Assay Kit (Beyotime Biotechnology, Shanghai, China). The loading capacity of nanoparticles was calculated according to the formula: loading capacity $=[($ Total OVA - Free OVA) / nanoparticles weight] x 100\%. And the encapsulation efficiency was calculated as the following formula: [(Total OVA - Free OVA) / Total OVA] x 100\%. 
The OVA release behavior from MCO NVs at $37{ }^{\circ} \mathrm{C}$ was evaluated through detection of antigens in dialysate after nanovaccines dialysis with 50-kDa MWCO dialysis tubing at preset time points.

\section{In vitro experiments}

\subsection{Cytotoxicity assessment}

Bone Marrow-Derived Dendritic Cells (BMDC) used for the in vitro experiments were obtained from femurs of C57BL/6 female mouse (Beijing WTLH Laboratory Animal Technology Co., Ltd, Beijing, China) as the described method. ${ }^{1}$ BMDCs were induced with complete RPMI 1640 medium containing 10\% fatal bovine serum (FBS), 10ng/mL IL-4 and 20ng/mL GM-CSF at $37^{\circ} \mathrm{C}$. On day 7 immature BMDCs were harvested to estimate the effect of MCO NVs on antigen present cells (APC).

To study the cytotoxicity of MCO NVs, BMDCs were seeded in 96-well plate and co-cultured with Free OVA, Free MPLA \& CpG ODN \& OVA (Free MCO) and MCO NVs at various concentrations for 24 hours, respectively. The cell viability was evaluated by Cell Counting Kit-8 (CCK-8, Dojindo Molecular Technologies, Inc., Japan) assay.

\subsection{Cellular uptake}

To assess the effect of MCO NVs on the cellular uptake of nanoparticles in BMDCs, OVA was labeled with Fluorescein Isothiocyanate (FITC). After co-incubated with FITC-labeled Free OVA, Free MCO or MCO NVs for 6 hours (OVA concentration was $10 \mu \mathrm{g} / \mathrm{mL}$ ), BMDCs were washed three times with PBS and fixed with 4\% paraformaldehyde (Beijing Solarbio Science \& Technology Co., Beijing, China). Then the Lyso-Tracker Red and DAPI were used to stain lysosome and nuclear, respectively. Finally, confocal laser scanning microscopy (CLSM, Zeiss LSM 800, Germany) and ImageJ software were used to image and analyze the ability of nanoparticle internalization in BMDCs, respectively.

\subsection{DCs maturation and activation}

BD Accuri ${ }^{\mathrm{TM}}$ C6 flow cytometer (BD Biosciences, San Jose, CA) was used to detect the maturation and activation of BMDCs. DCs were incubated with different treatments for 8 hours (OVA concentration was $10 \mu \mathrm{g} / \mathrm{mL}$ ). Some BMDCs were stained 
with Cy5.5-labled anti-CD11c, APC-labeled anti-MHC-I and FITC-labeled anti-MHCII antibodies to evaluate the expression of main stimulating molecule MHC-I/II on BMDCs. Other BMDCs were stained with florescence labeled CD11c, CD40 and CD86 antibodies to study the co-stimulating molecules on BMDCs. Furthermore, the cytokines Interleukin-6 (IL-6) and tumor necrosis factor (TNF- $\alpha$ ) in the supernatant were detected by enzyme-linked immunosorbent assay (ELISA) kits (Thermo Fisher Scientific Inc., USA) to analyze whether BMDCs could be activated by MCO NVs.

\section{In vivo experiments}

\subsection{Sustained release of nanoparticles at injection site}

To observe the depot effect of MCO NVs in vivo, OVA (the model antigen) were labeled with fluorescent dye Cy7 before MCO NVs were prepared. Then C57BL/6 mice were injected subcutaneously with various formulations (Free Cy7-labeled OVA as control, the dosage of OVA was $40 \mu \mathrm{g}$ ). Fluorescence intensity at injection site was detected with in-vivo imaging system (CRi Maestro EX, USA) at different time points after injection.

\subsection{Tumor challenge and immunization}

All animals were treated under guidelines approved by the animal care and use committee at Peking Union Medical College, China. The 6-week-female C57BL/6 mice were injected with $5 \times 10^{5}$ E.G7-OVA tumor cells. When the tumor volume reached about $50 \mathrm{~mm}^{3}$, the mice were immunized with PBS, Free OVA, Free MCO and MCO NVs $(\mathrm{n}=6$, the content of OVA was $40 \mu \mathrm{g} /$ mouse) and all mice were immunized 3 times with an interval of one week. To estimate the therapeutic effect of MCO NVs in vivo, the tumor size and survival period were recorded.

To evaluate the immune responses, mice were immunized 3 times as described above and sacrificed on $3^{\text {rd }}$ day after the last immunization. The tissue of tumor, spleen and lymph nodes were harvested for the following analysis of immune responses.

\subsection{Antibody secretion}

The blood was collected from the ophthalmic vein and centrifuged twice at $3000 \times \mathrm{rpm}$ for $20 \mathrm{~min}$ to obtain the serum before the mice were sacrificed. The concentration of OVA-specific IgG, IgG1 and IgG2a in serum were quantified using 


\section{ELISA kit.}

\section{4 $\mathrm{T}$ cell immune response}

Lymph nodes and spleens were collected from immunized mice and grinded into single cell suspension. Lymphocytes were separated with the Lymphocyte Separation Kit (Beijing Solarbio Science \& Technology Co., Beijing, China) and stained with fluorescence-labeled antibodies of CD3, CD4 and CD8.

To further access the antigen-specific $\mathrm{T}$ cell immune responses, $1 \times 10^{6}$ spleen lymphocytes were incubated with OVA $(20 \mu \mathrm{g} / \mathrm{mL})$ for $72 \mathrm{~h}$, and then stained with CD3, CD8 and SIINFEKL antibodies.

\subsection{Cytokine secretion}

$2.5 \times 10^{5}$ spleen lymphocytes from immunized mice were seeded into 96-well plates and incubated with OVA $(20 \mu \mathrm{g} / \mathrm{mL})$ for $48 \mathrm{~h}$. After $450 \mathrm{~g}$ centrifugation, supernatant was collected to measure cytokine level of IL-12, INF- $\gamma$, IFN- $\alpha$ and IFN- $\beta$ using ELISA kit.

\subsection{Memory $T$ cells}

Spleen lymphocytes were separated, collected and seed into 24-well plates. After incubation with OVA $(20 \mu \mathrm{g} / \mathrm{mL})$ for 72 hours, the cells were labeled with CD4, CD8, CD44, CD62L antibodies. Flow cytometer was used to detect the proportion of memory T cells.

\subsection{Lymphocyte proliferation}

Spleen lymphocytes were re-stimulated with OVA $(20 \mu \mathrm{g} / \mathrm{mL})$ for $48 \mathrm{~h}$ and stained with antibodies of CD3, CD4, CD8 and Ki67, then T cell proliferation was measured by flow cytometer.

\subsection{Pathological analysis}

Tumor issues collected from mice treated with various formulations were fixed using paraformaldehyde and dehydrated before embedding into paraffin. The slices of tumor $(5 \mu \mathrm{m})$ were stained with hematoxylin-eosin $(\mathrm{H} \& \mathrm{E})$ and images were acquired with fluorescence microscope (Olympus BX53, Japan) for pathological analysis.

\section{Signal transduction pathways}

To assess the activation of nuclear factor-kappa B (NF-kB), p65 was detected 
through immunofluorescence staining with NF-kB Activation-nuclear Translocation Assay Kits (Beyotime Biotechnology, China). Briefly, after incubation with PBS, Free MCO or MCO NVs for 24 hours, BMDCs were washed with PBS and fixed with Immunol Staining Fix Solution (Beyotime Biotechnology, Shanghai, China). NF-кB p65 antibodies were incubated $1 \mathrm{~h}$ and washed three times using washing solution to remove the unbound antibodies, then $\mathrm{Cy} 3$ fluorescein-conjugated secondary antibody were incubated $1 \mathrm{~h}$ and washed three times again. At last, nuclear was stained with DAPI before CLSM was used to observe the location of P65.

After BMDCs were treated with different formulations for $24 \mathrm{~h}$, proteins were extracted using Cell Lysis Buffer for Western and IP (Beyotime Biotechnology, Shanghai, China) and then separated by polyacrylamide gel electrophoresis and transferred into polyvinylidene fluoride (PVDF) membrane. Then solution containing $5 \%$ bovine serum albumin (BAS) was used to seal PVDF membrane for $2 \mathrm{~h}$ at room temperature. Afterwards, membranes were incubated with primary antibodies of $\mathrm{p}-\mathrm{JNK}$, p-p38, p-ERK, p-IRF3 and GAPDH (Cell Signaling Technology, USA) for overnight at $4{ }^{\circ} \mathrm{C}$, and then second antibodies reacted for $1 \mathrm{~h}$ at room temperature. Finally, PVDF membrane was developed by Immobilon ECL Ultra Western HRP Substrate (Millipore, USA) and exposed using fluorescence chemiluminescence imaging system (Clinx Science Instruments Co., Ltd, Shanghai, China). Image J software were used to analyze the gray value of stripe.

\section{Statistical Analysis}

The data were displayed as mean \pm standard deviation (SD). Student's $t$ test and ANOVA were used to detect the statistical differences among the groups. P value no more than 0.05 was considered significant difference. 


\section{Supplementary Tables and Figures}

Table S1. The size, PDI and Zeta potential of MC NPs and MCO NVs.

\begin{tabular}{cccc}
\hline NPs & Size $(\mathrm{nm})$ & PDI & Zeta potential $(\mathrm{mV})$ \\
\hline MC NPs & $142.2 \pm 0.9$ & $0.1 \pm 0.02$ & $-25.7 \pm 0.3$ \\
MCO NVs & $159.1 \pm 0.6$ & $0.2 \pm 0.005$ & $-20.8 \pm 1.2$ \\
\hline
\end{tabular}

Data were expressed in the form of mean $\pm S D(n=6)$

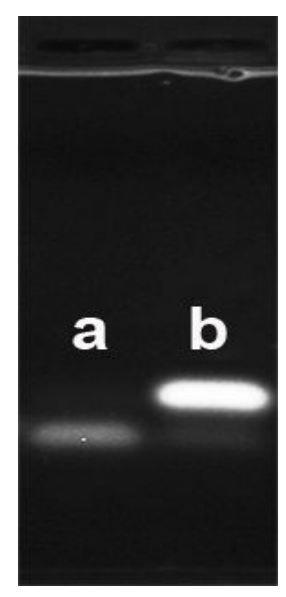

Figure S1. The typical agarose gel electrophoresis of MPLA-CpG (a: CpG ODN, b: MPLA-CpG) 


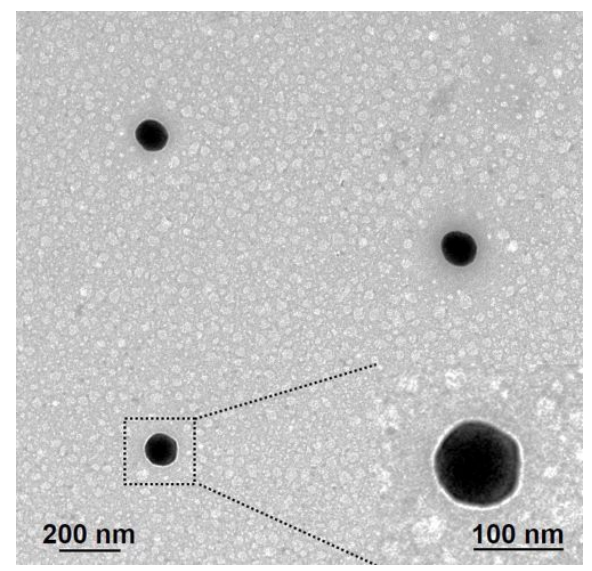

Figure S2. The representative TEM image of MPLA-CpG nanoparticles.

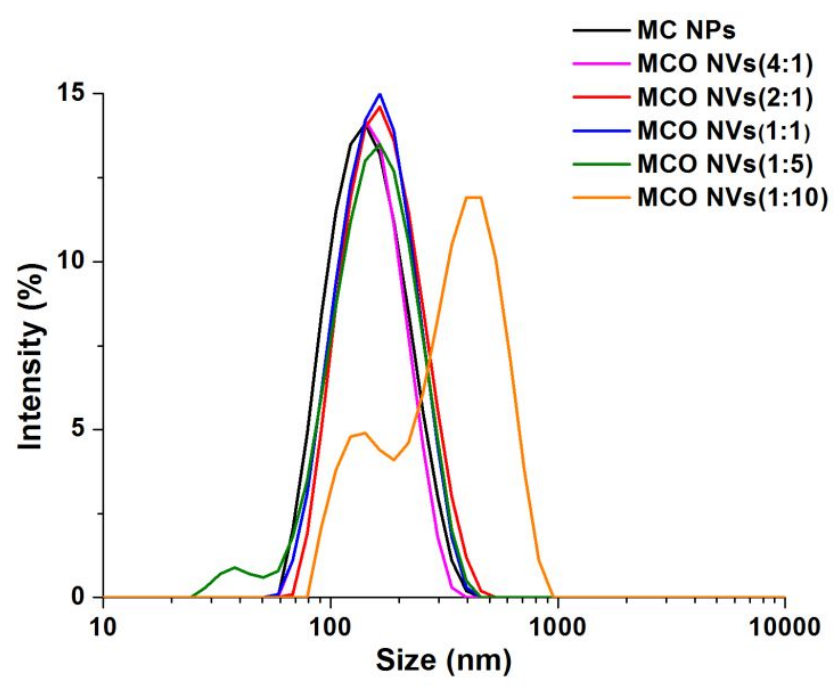

Figure S3. The size distribution of MC NPs and MCO NVs prepared with various molar ratio. 


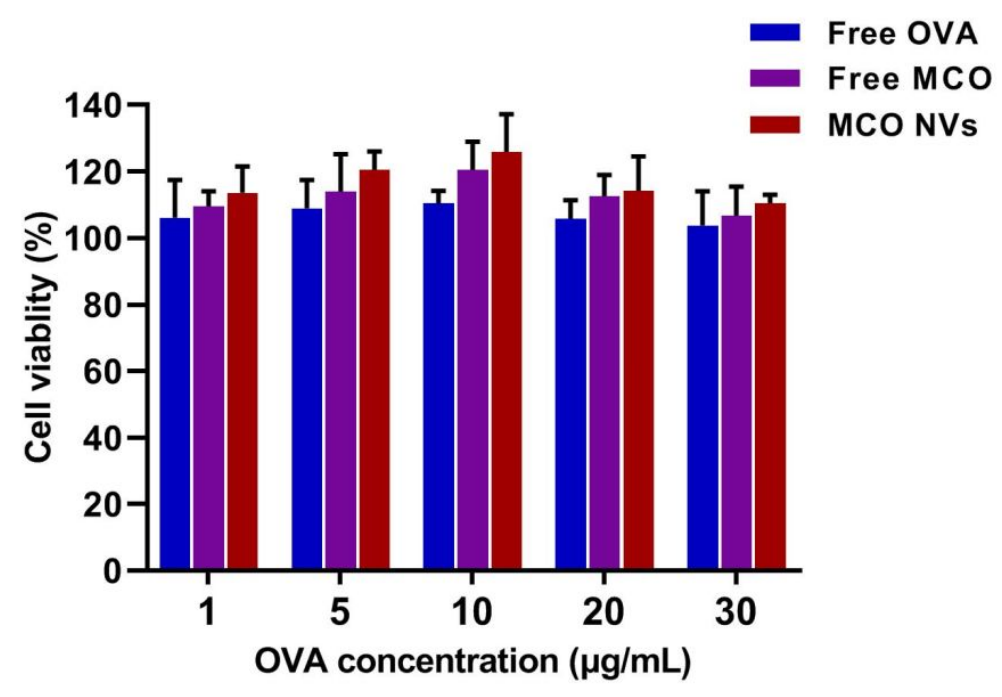

Figure S4. The cell viability of BMDCs treated with various formulations for $24 \mathrm{~h}$ was detected by Cell Counting Kit-8 (CCK-8) assay. PBS treated group was used as the control and the cell viability was considered as $100 \%$. Data were expressed in the form of mean $\pm \operatorname{SD}(n=6)$.

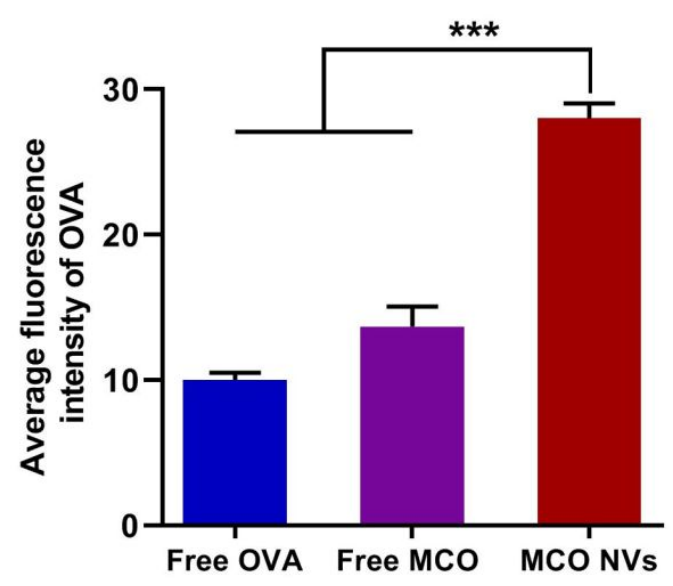

Figure S5. Quantitative analysis of average fluorescence intensity of OVA in BMDCs co-incubated 6 h with FITC-labeled Free OVA, Free MCO and MCO NVs. Data were expressed in the form of mean $\pm \mathrm{SD}(\mathrm{n}=3) . * * * P<0.001$. 


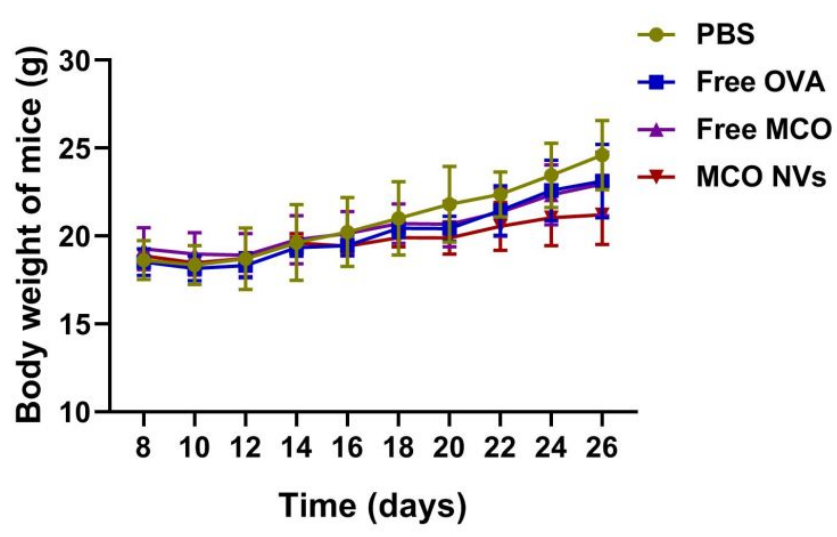

Figure S6. The body weight curves of mice treated with different formulations. Data were expressed in the form of mean \pm SD $(n=6)$. No significant difference was observed among these groups $(P>0.05)$.
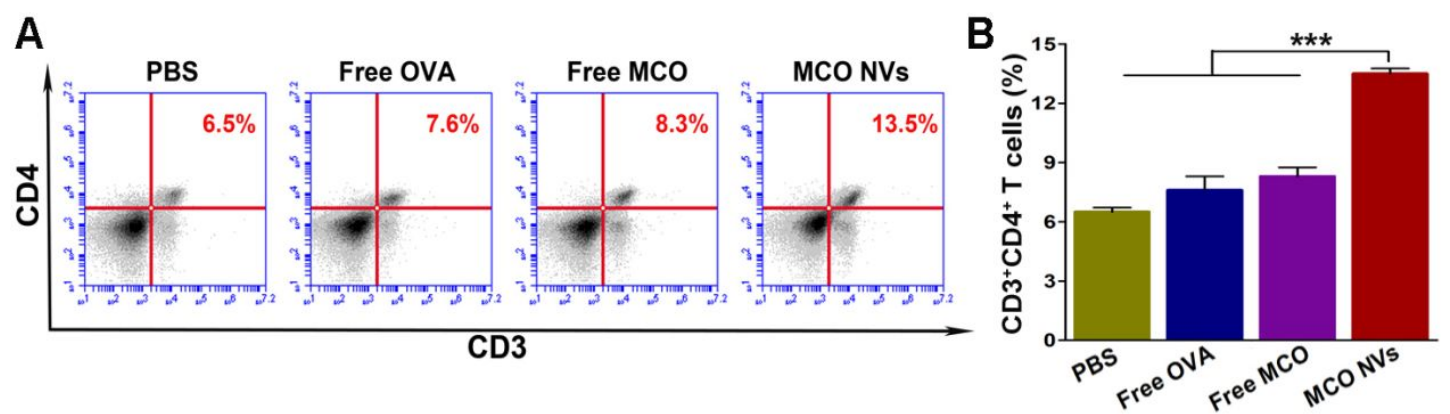

Figure S7. Representative FACS plots (A) and histogram of percentage (B) of $\mathrm{CD}^{+} \mathrm{CD}^{+} \mathrm{T}$ cells in spleen collected from mice treated with various formulations.

Data were expressed in the form of mean $\pm \mathrm{SD}(\mathrm{n}=6) .{ }^{*} * * P<0.001$. 

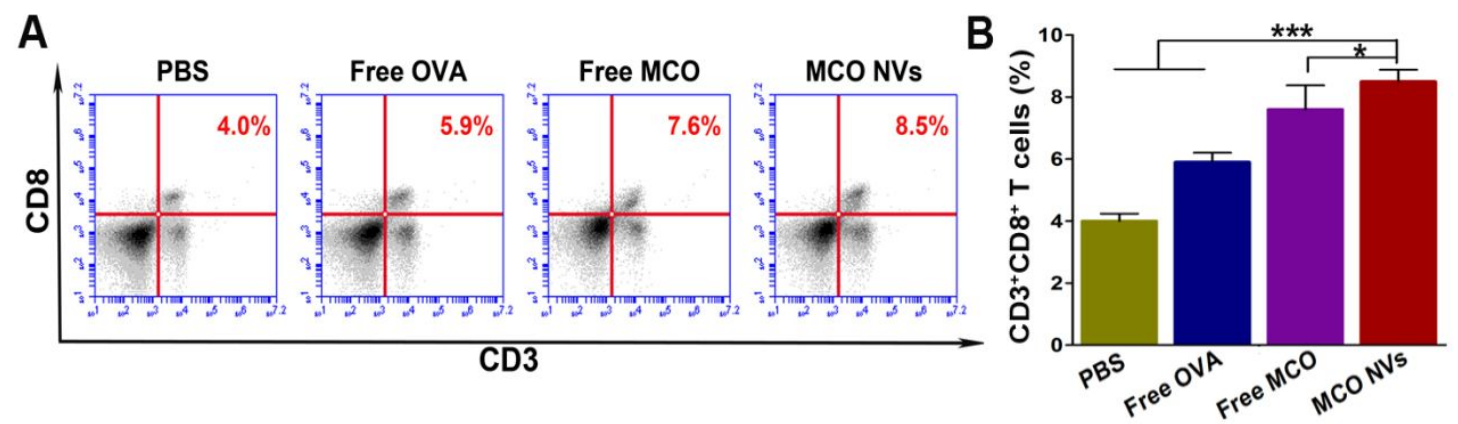

Figure S8. Representative FACS plots (A) and histogram of percentage (B) of $\mathrm{CD}^{+} \mathrm{CD}^{+} \mathrm{T}$ cells in spleen collected from mice treated with various formulations. Data were expressed in the form of mean $\pm \mathrm{SD}(\mathrm{n}=6) .{ }^{*} P<0.05, * * * P<0.001$.

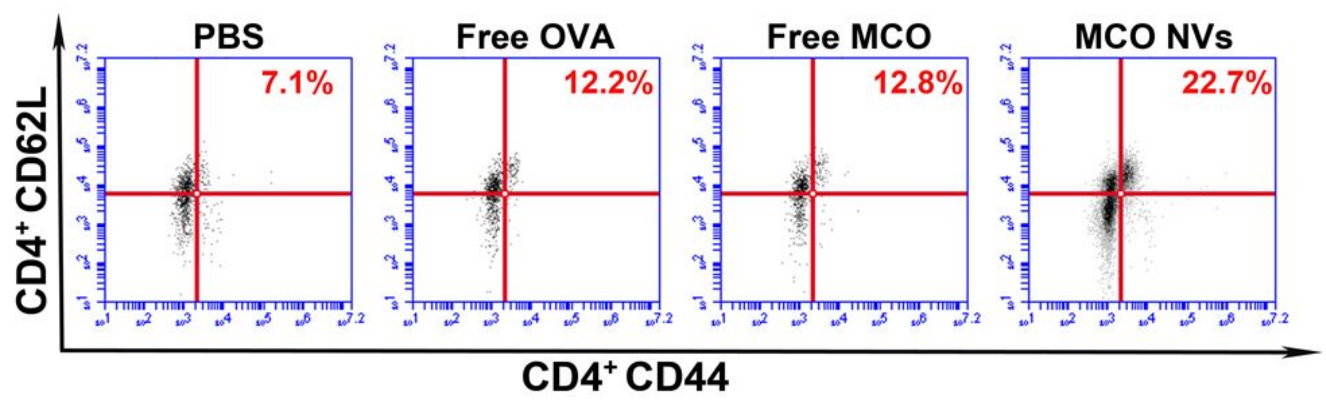

Figure S9. Representative FACS plots of $\mathrm{CD}^{+} \mathrm{T}$ memory cells in spleen.

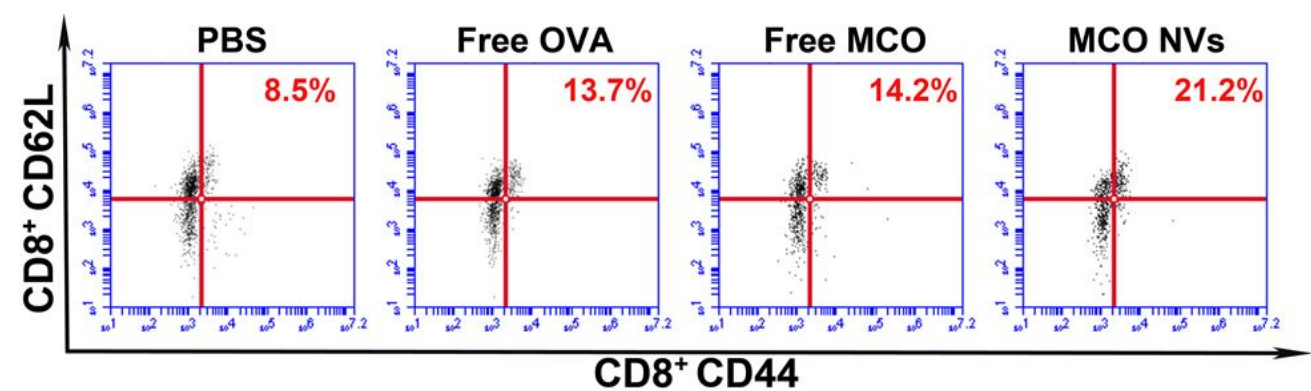

Figure S10. Representative FACS plots of $\mathrm{CD} 8^{+} \mathrm{T}$ memory cells in spleen. 

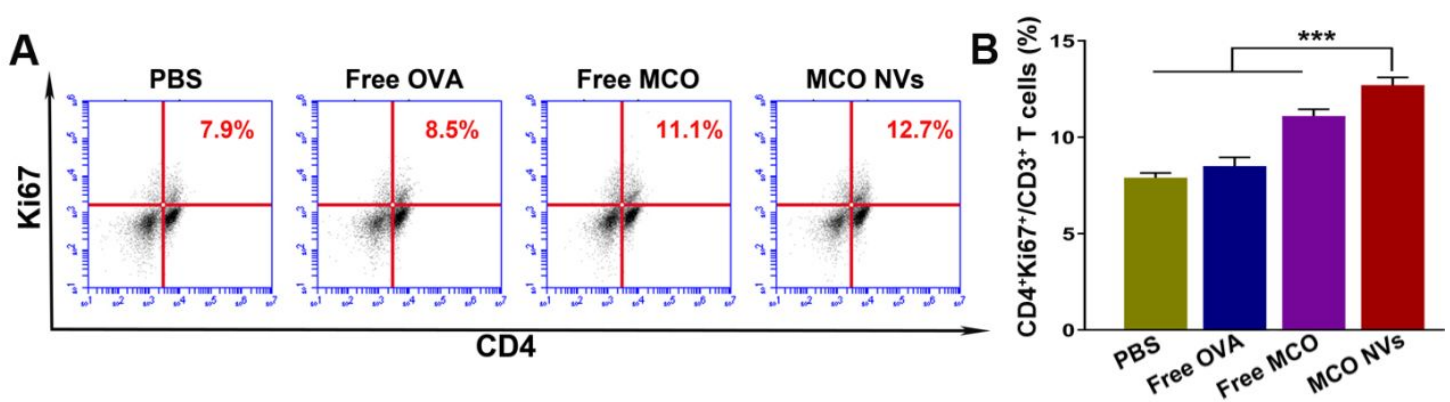

Figure S11. The proliferation of $\mathrm{CD}^{+} \mathrm{T}$ cells was detected after spleen lymphocytes from immunized mice with various formulations were re-stimulated with OVA for 48 h. (A) FACS plots and (B) the histogram of percentage of $\mathrm{CD}^{+} \mathrm{ki}^{+} 7^{+} \mathrm{T}$ cells. Data were expressed in the form of mean $\pm \operatorname{SD}(n=6)$. ${ }^{*} * * P<0.001$.
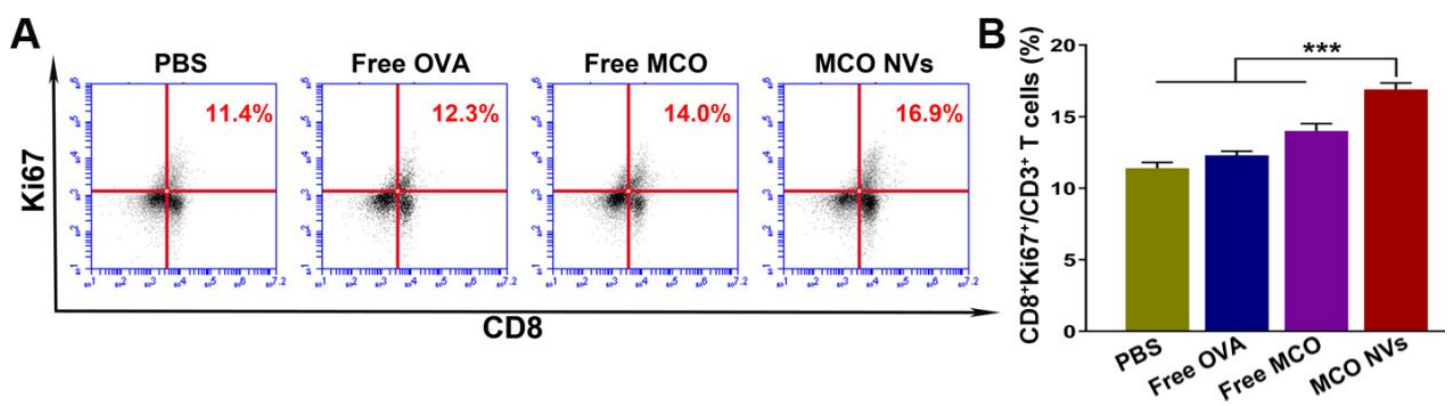

Figure S12. The proliferation of $\mathrm{CD}^{+} \mathrm{T}$ cells was detected after spleen lymphocytes from immunized mice with various formulations were re-stimulated with OVA for 48 h. (A) FACS plots and (B) the histogram of percentage of $\mathrm{CD} 8^{+} \mathrm{ki} 67^{+} \mathrm{T}$ cells. Data were expressed in the form of mean $\pm \operatorname{SD}(n=6)$. $* * * P<0.001$. 

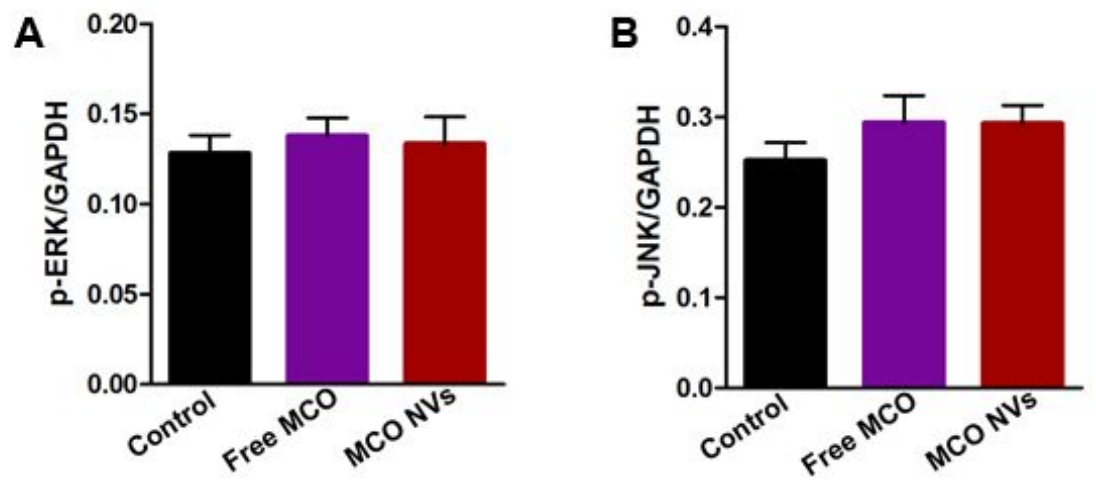

Figure S13. The relative gray values of p-ERK (A) and p-JNK (B) compared to internal parameter of GAPDH were calculated and showed no statistic difference among all groups. Data were expressed in the form of mean $\pm \operatorname{SD}(n=6)$.

\section{Reference}

(1) Sheng, K. C.; Kalkanidis, M.; Pouniotis, D. S.; Esparon, S.; Tang, C. K.; Apostolopoulos, V.; Pietersz, G. A., Delivery of Antigen Using a Novel Mannosylated Dendrimer Potentiates Immunogenicity in Vitro and in Vivo. Eur. J. Immunol. 2008, 38 (2), 424-436. 